\title{
Percutaneous image-guided double oblique anterior approach to the acetabulum for cementoplasty
}

\author{
J. Garnon ${ }^{\text {a, b, *, P. De Marini }}{ }^{\text {a }}$, L. Meylheuc ${ }^{\text {b }}$, D. Dalili ${ }^{\text {c,d }}$, R.L. Cazzato ${ }^{\text {a }}$, \\ B. Bayle ${ }^{\mathrm{b}}, \mathrm{A}$. Gangi ${ }^{\mathrm{a}}$ \\ a Department of Interventional Radiology, Nouvel Hôpital Civil, 1, Place de L'Hôpital, 67096 Cedex, Strasbourg, France \\ ${ }^{\mathrm{b}}$ UMR 7357 CNRS, ICube, INSA Strasbourg, Université de Strasbourg, 67091, Strasbourg, France \\ ${ }^{\text {c } N u f f i e l d ~ O r t h o p a e d i c ~ C e n t r e, ~ O x f o r d ~ U n i v e r s i t y ~ H o s p i t a l s ~ N H S ~ F o u n d a t i o n ~ T r u s t, ~ W i n d m i l l ~ R d, ~ O x f o r d, ~ O X 3 ~ 7 L D, ~ U K ~}$ \\ d School of Biomedical Engineering and Imaging Sciences, King's College London, London, UK
}

AIM: To report the technique of percutaneous double oblique anterior access to the ace tabulum and evaluate its feasibility and safety.

MATERIALS AND METHODS: Pelvic computed tomography (CT) examinations of 60 patients ( 30 men and 30 women; mean age $62.6 \pm 13.2$ years) were retrieved randomly from the picture archiving and communication system (PACS). A virtual intraosseous trajectory was defined on both sides on the axial CT images with the entry point marked $1 \mathrm{~cm}$ above the anterosuperior iliac spine and the endpoint defined just above the level of the ischial spine at the midpoint of the posterior acetabulum. Patient age, sagittal oblique angulation, axial oblique angulation, length of intraosseous trajectory, distance from the hip joint, thickness of the iliac bone cortex, and intervening structure(s) between the skin and the bone entry points were recorded.

RESULTS: The mean sagittal and axial oblique angulations were $34.2 \pm 4.5^{\circ}$ and $31.5 \pm 6.7^{\circ}$, respectively, and mean length of the intraosseous trajectory was $11.8 \pm 0.9 \mathrm{~cm}$. The axial oblique angle and length of the intraosseous trajectory were significantly lower in the female than the male population $(p<0.05)$. None of the virtual trajectories traversed the hip joint. In $112 / 120$ trajectories (93.3\%), there were no cortical breaches in the iliac bones. In eight trajectories in four patients, the virtual trajectory crossed either the medial iliac cortex $(4 / 120 ; 3.3 \%)$ or the medial iliac cortex and the iliacus muscle (4/120; 3.3\%).

CONCLUSION: The anterosuperior iliac and ischial spines can be used safely and reliably as landmarks to perform the double oblique anterior approach. 


\section{Introduction}

Percutaneous cementoplasty of acetabular osteolysis was described $>25$ years ago. ${ }^{1}$ The technique is effective not only in alleviating pain generated from malignant bony infiltration, but also to provide mechanical stability through consolidation in cases of bone destruction. ${ }^{2,3}$ Most of the published literature reports a direct axial approach to the roof of the acetabulum via anterior or lateral access, using fluoroscopy, computed tomography (CT), or a combination of both. ${ }^{4,5}$ In the case of extensive osteolysis with multiple osteolytic areas, most of the publications advocate the use of multiple needles to inject as much cement as possible around the hip. ${ }^{2,6,7}$ An alternative technique is to approach the lesion(s) along the long axis and continuously inject cement through a single puncture site whilst progressively withdrawing the bone trocar. ${ }^{8}$ For the pelvic bone, the retrograde trans-pubic and ascending trans-ischial approaches have been described to access the anterior and posterior acetabulum, respectively, along their long axis. ${ }^{9,10}$ In an attempt to fill the osteolytic space involving both the acetabular roof and the posterior acetabulum with a single anterior puncture, an alternative double oblique anterior trans-iliac approach may be used but has not yet been standardised in the literature. ${ }^{8}$ The purpose of the present study was to present the technique implementing a double oblique anterior approach to the acetabulum based on standardised anatomical landmarks, as well as evaluate its feasibility and safety.

\section{Materials and methods}

This was a single centre observational study. Institutional review board approval was obtained for the retrospective evaluation of patient radiological data and post-processing.

\section{Double oblique anterior approach}

The principle of the approach is to enter into the iliac bone at a point situated $1 \mathrm{~cm}$ above the anterosuperior iliac spine (ASIS). The bone trocar (Special vertebroplasty needle, Optimed, Germany) is then advanced through the iliac wing whilst applying craniocaudal and lateromedial angulation above the roof of the acetabulum, until the tip of the needle reaches the posterior acetabulum at the most superior part of the ischial spine. The typical trajectory for the anterior double oblique percutaneous approach is presented on a pelvic model in Fig 1. A clinical example for acetabular cementoplasty is shown in Fig 2.

The approach can be performed with standalone fluoroscopic guidance and is presented in detail in Fig 3. The

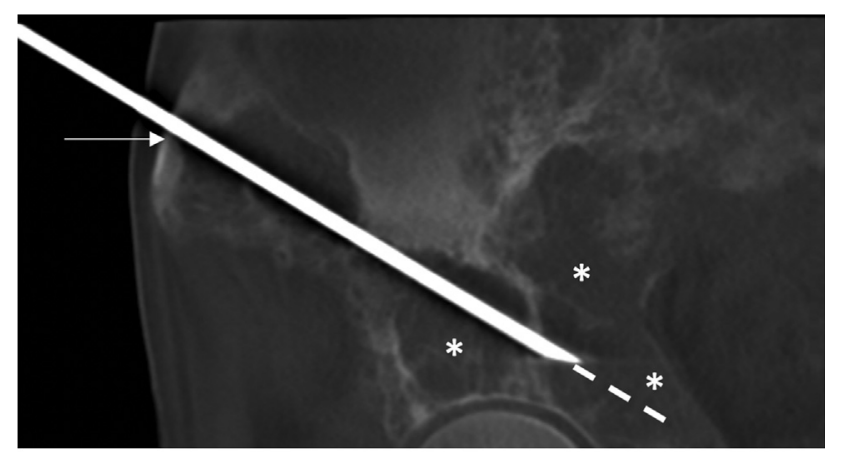

(a)

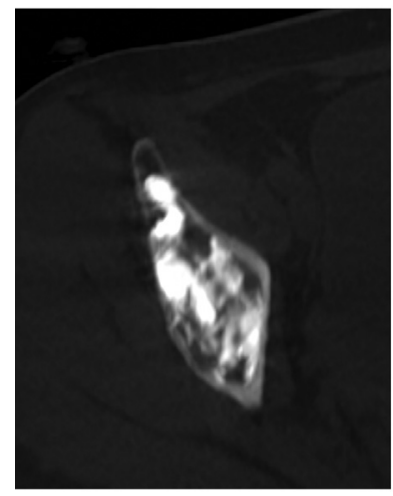

(b)

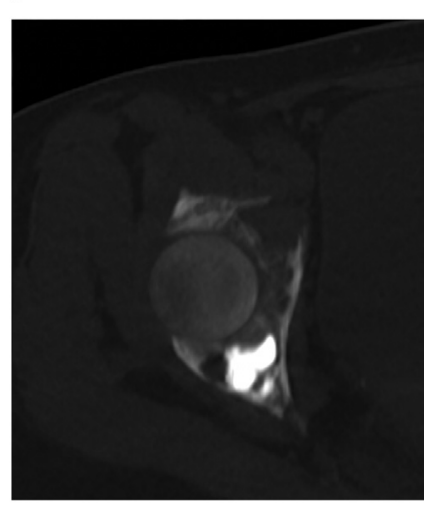

(c)
Figure 2 Double oblique approach for percutaneous cementoplasty. (a) The bone trocar is inserted anteriorly in the iliac wing (arrow) and is advanced towards the posterior acetabulum (dotted line), allowing it to enter the long axis of a large osteolytic metastasis (asterisks). (b) Cement within the acetabular roof and (c) the posterior acetabulum with a single access.

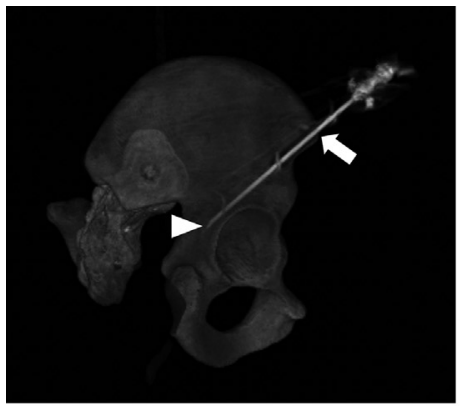

(a)

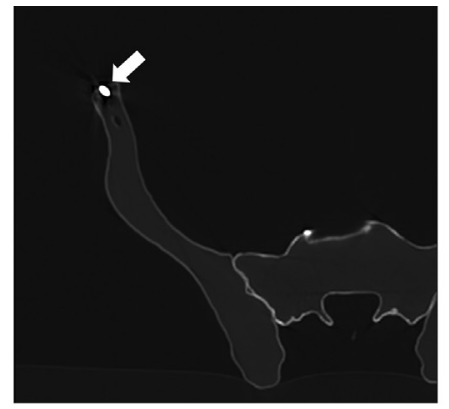

(b)

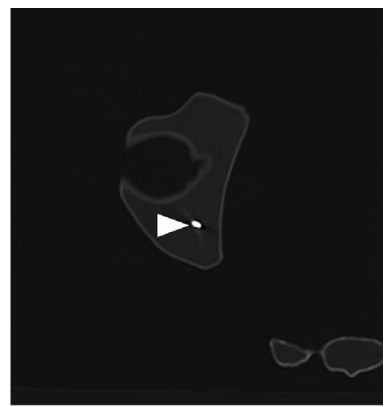

(c)

Figure 1 Double oblique anterior approach to the acetabulum. (a) VRT (right lateral view) and (b,c) axial CT image illustrating the double oblique approach in a phantom. The needle enters the iliac wing (arrow in a,b) just above the ASIS and is advanced medially and caudally to the superior part of the posterior acetabulum (arrowhead in a,c). 


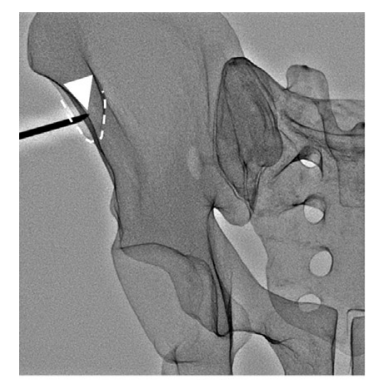

(a)

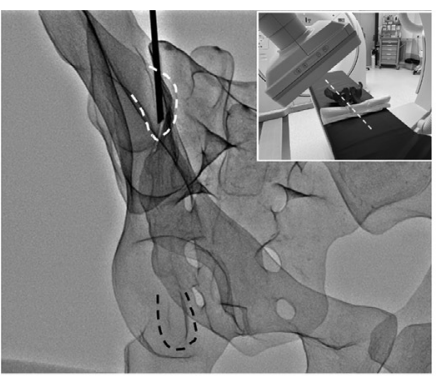

(b)

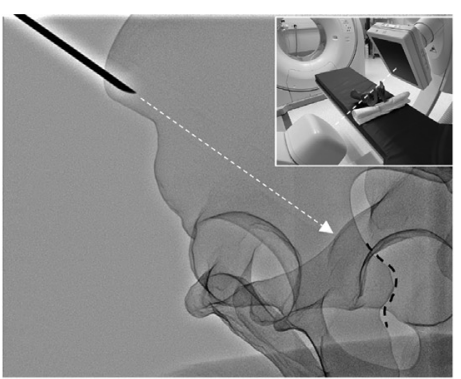

(c)

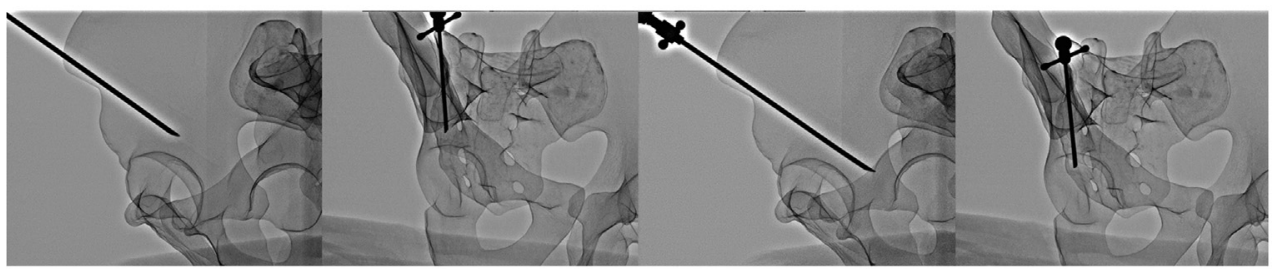

(d)

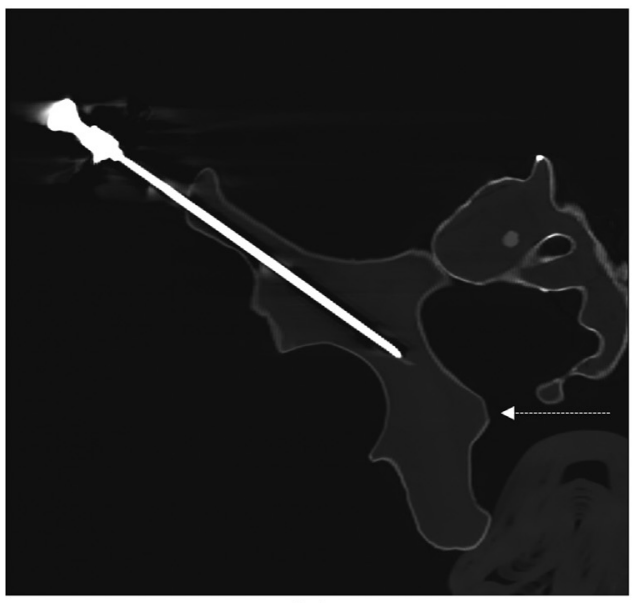

(e)

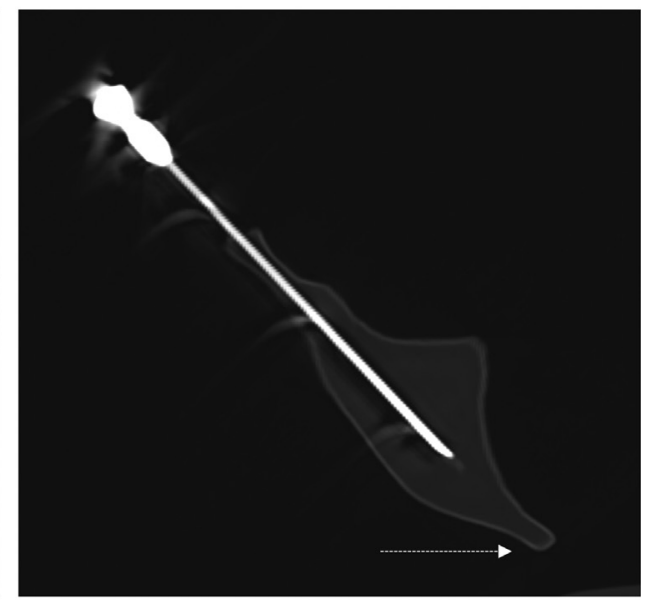

(f)

Figure 3 Approach with fluoroscopic guidance. (a) Anteroposterior projection: the middle part of the iliac crest (arrowhead) just above the ASIS (dotted line) is located. (b) The $\mathrm{C}$ arm is rotated to align with the needle inserted in the mid point of the iliac crest (white dotted line) with the ischial spine (black dotted line). (c) The $C$ arm is rotated perpendicular to the oblique view, which displays the optimal projection to reach the posterior acetabulum without transgressing the joint (dotted arrow). The ischial spine is also visible (black dotted line). (d) The needle is advanced under alternative perpendicular oblique views to ensure that the tip targets the ischial spine on the entry point view and the cau docranial angulation is appropriate in the progression view. Note that this approach enables reaching the roof of the acetabulum and the posterior acetabulum depending on the depth of the needle. Corresponding (e) sagittal oblique and (f) axial oblique CT images illustrating the intraosseous trajectory of the double oblique approach with the ischial spine as the target landmark (dotted line).

same access can be performed with cone-beam (CB)CT or $\mathrm{CT}$ guidance with a needle trajectory software using the same aforementioned landmarks (Fig 4). Under CBCT guidance, the needle is then advanced using both the entry point and progression views ${ }^{11}$ to check that the trajectory is correct. Utilising CT guidance, the application of quick volumetric acquisition with post-processing multiplanar reconstructions (MPR) is a precise way to assess correct needle advancement. ${ }^{12}$

\section{Evaluation of the feasibility and safety of the approach}

To analyse the feasibility of the double oblique approach, pelvic CT images of 60 patients ( 30 men and 30 women) were retrieved randomly from the picture archiving and communication system (PACS). DICOM images were analysed on the bone window presets by an interventional radiologist with 10 years of experience in musculoskeletal (MSK) interventions. A virtual trajectory was defined on axial CT images using the aforementioned technique: the entry point was marked $1 \mathrm{~cm}$ above the ASIS and the endpoint was defined just above the level of the ischial spine in the midpoint of the posterior acetabulum (Fig $5 \mathrm{a}-\mathrm{c}$ ).

\section{Data collection and analysis}

The following data were recorded (Fig 5d,e): (a) patient age; (b) sagittal oblique angulation; (c) axial oblique angulation; (d) length of intraosseous trajectory, (e) breach 


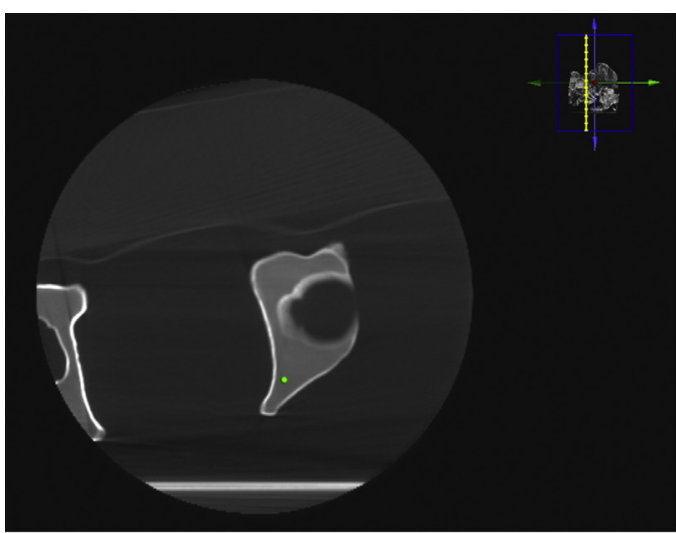

(a)

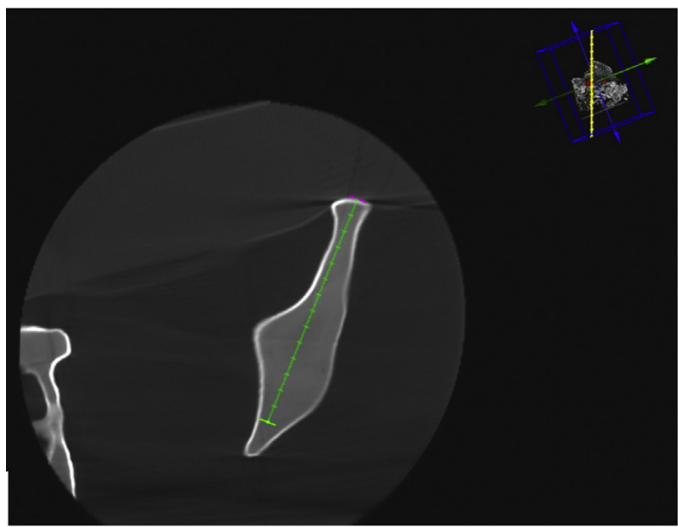

(c)

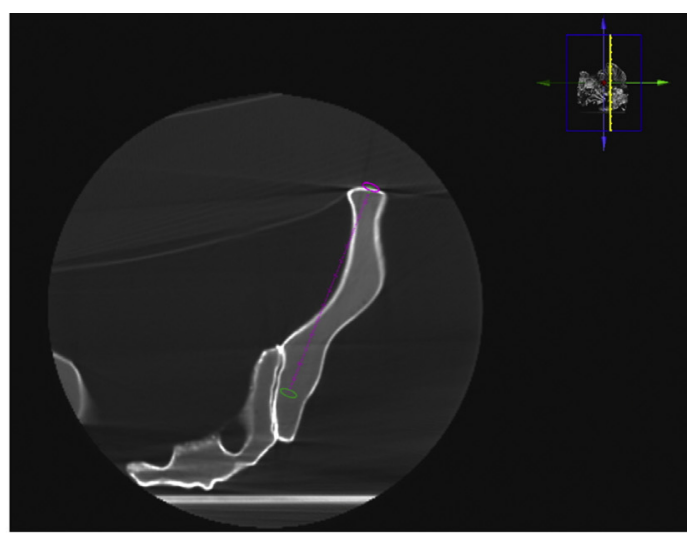

(b)

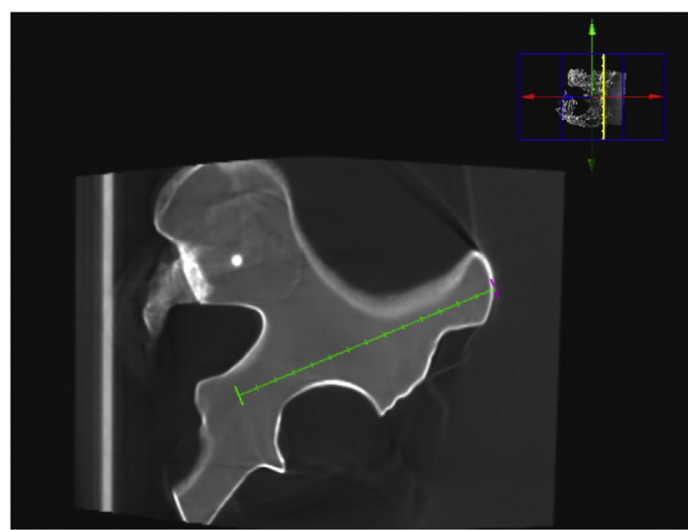

(d)

Figure 4 Approach with CBCT guidance and needle trajectory. (a) The target point is marked in the posterior acetabulum at the level of the upper part of the ischial spine. (b) The entry point is defined $1 \mathrm{~cm}$ above the ASIS. (c) Planned needle trajectory. (d) Progression view.

of the hip joint; ( $f$ ) disruption of the cortices (medial, lateral or both) of the iliac bone, (g) any intervening structure(s) between the skin and the bone entry points. For each patient both hemipelvices were analysed, resulting in a total of 120 analyses.

Descriptive statistics were used to present results. Categorical variables were expressed as absolute numbers and percentages. Continuous variables were expressed as means with standard deviations and ranges. Difference attributed to age, length, or pelvic angles between genders were assessed using a $t$-test. A p-value of $<0.05$ was considered statistically significant. Statistical analysis was performed using R v3.6.3 (R Foundation for Statistical Computing, Vienna, Austria).

\section{Results}

Mean patient age was $62.6 \pm 13.2$ (range $22-86$ ) years. There was no significant difference of age between men $(62.7 \pm 12$ years) and women $(62.4 \pm 14.5$ years; $p=0.92)$. Mean sagittal and axial oblique angulations were $34.2 \pm 4.5^{\circ}$ (range $24-46^{\circ}$ ) and $31.5 \pm 6.7^{\circ}$ (range $15-48^{\circ}$ ), respectively. Mean length of the intraosseous trajectory was $11.8 \pm 0.9$ (range 9.5-14) $\mathrm{cm}$. The axial oblique angle and length of trajectory were significantly lower in the female than male population: $29.6 \pm 7.5$ versus $33.4 \pm 5.3^{\circ} \quad(p=0.002)$ and
$11.4 \pm 0.8$ versus $12.1 \pm 0.9 \mathrm{~cm}(p<0.001)$, respectively. There was no significant difference between the two populations for the sagittal oblique angle.

None of the virtual trajectories crossed the hip joint. In $112 / 120$ trajectories (93.3\%), there was no transgression of the cortex of the iliac bone. In two female patients, the virtual trajectory intersected the medial iliac cortex on both sides $(4 / 120 ; 3.3 \%)$. In two other female patients, the virtual trajectory intersected the medial iliac cortex and the iliac muscle on both sides (4/120, 3.3\%; Fig 6). Intervening structures between the skin and the iliac bone included abdominal wall muscles in 17/120 trajectories (14.2\%) and abdominal wall muscles plus digestive structure(s) in 3/120 trajectories (2.5\%). All soft-tissue structures were deemed easily manually displaceable. In the remaining 100 trajectories, subcutaneous fat was the only structure interposed between the skin and the bone entry points.

\section{Discussion}

The present study describes the anatomical landmarks for the double oblique intraosseous approach to the acetabulum. It allows rapid and consistent identification of the entry point and the target point when using needle trajectory software. Knowledge of the respective fluoroscopy 


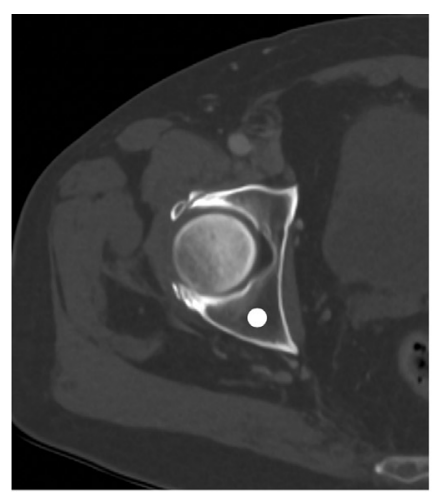

(a)

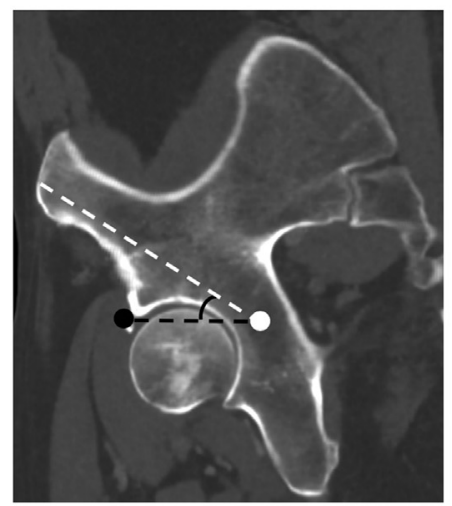

(d)

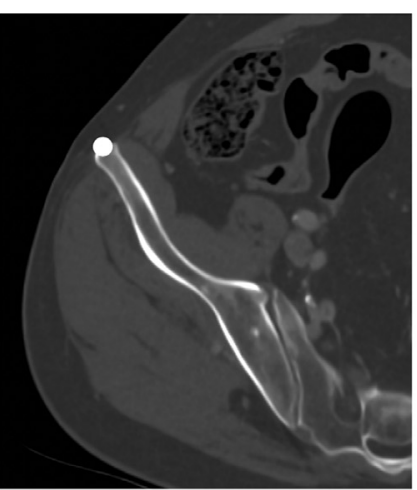

(b)

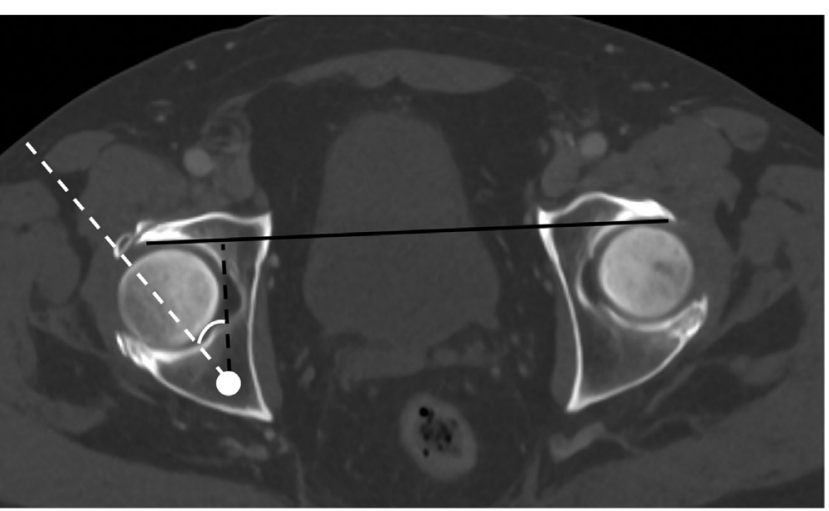

(e)

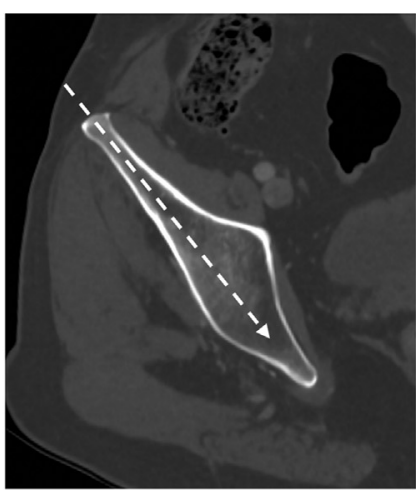

(c)

Figure 5 Imaging study. (a) Target point (circle) marked just above the level of the ischial spine in the mid point of the posterior acetabulum. (b) Entry point (circle) marked $1 \mathrm{~cm}$ above the ASIS in the mid point of the anterior iliac wing. (c) Virtual planned trajectory (dotted arrow): no intervening structure, no transgression of the iliac cortex. (d) Sagittal oblique view: the virtual trajectory (dotted line) does not transfix the joint until it reaches the target point (white circle). The sagittal angulation is measured between the virtual trajectory and the line (black dotted line) joining the target point and the anterior part of the acetabulum (black circle). (e) The axial angulation is measured on an axial view between the virtual trajectory and the sagittal axis of the bony pelvis.

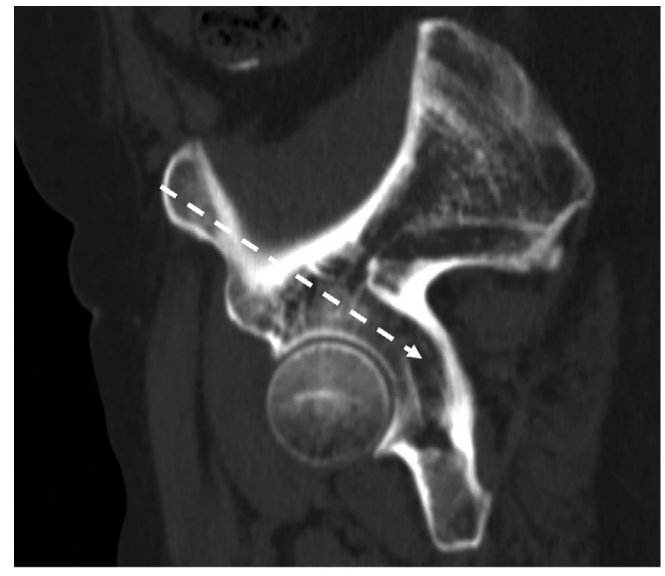

(a)

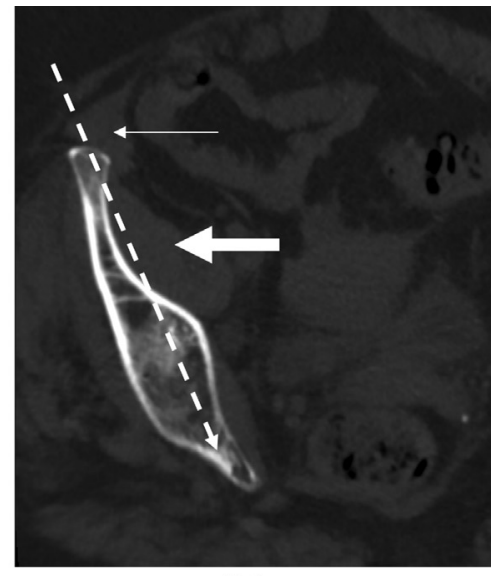

(b)

Figure 6 Imaging study: case of a failed virtual approach using the anatomical landmarks. (a) Sagittal oblique showing the virtual trajectory (dotted arrow). (b) Axial oblique in the axis of the planned trajectory: the access transfixes the abdominal muscle (thin arrow, easily removable by displacing the abdominal apron), the medial cortex and the iliac muscle.

landmarks is also noteworthy as appropriate angulation is immediately estimated without the need to repeat multiple three-dimensional acquisitions during needle advancement in case of doubt. Finally, access can be performed with standalone fluoroscopic guidance should the important obliquity preclude the use of a planned trajectory as encountered several times based on the authors' clinical experience. 
Based on this imaging study, the double oblique approach seems feasible in almost all cases. Imaging findings demonstrate that the access defined purely on two anatomical landmarks still carries a low risk of breach of the medial iliac cortex in women. Routine careful review of the diagnostic CT images or of the planned trajectory prior to needle insertion is required in order to adjust the access whenever necessary to mitigate against any cortical transgression during advancement of the needle.

Regarding safety, the major theoretical concern may be the risk of injury to the abdominal muscles or to the abdominal viscus in obese patients. In the authors' clinical practice using this approach, the abdominal muscles and the digestive structures are easily avoidable by physically displacing the abdominal apron towards the contralateral side. Hence, the access does not traverse any muscles before entering the iliac bone, thereby theoretically reducing the risk of haematoma formation., ${ }^{9,13,14}$ Additionally, this approach avoids the emergence of the lateral cutaneous nerve of the thigh that courses in the subcutaneous fat below the level of the ASIS. The intraosseous trajectory is relatively long but remains feasible in clinical practice.

This approach can be valuable when performing cementoplasty in patients with extensive disease. In the authors' clinical experience, the posterior acetabulum could be filled whilst still gaining access to the acetabular roof through a single access point by simply withdrawing the bone trocar during cement injection. It is advantageous compared to a pure axial anterior access, for which diffusion of cement to the posterior column is unpredictable and limited most of the time as the tip of the needle lies away from the ischial spine. This is noteworthy as cement repartition in the different osteolytic areas of the acetabulum has been identified as a prognostic factor for reducing the incidence or worsening of a pathological fracture. ${ }^{6,15}$ It may also avoid for the need of patient repositioning to access specifically the posterior acetabulum through a lateral or posterior approach. Finally, the double oblique approach might decrease the risk of softtissue cement leakage along the needle tract because of the long-intraosseous tunnel, which allows better control of cement backflow. It is, however, difficult to prove the superiority of the aforementioned access over a direct approach scientifically without a dedicated randomised controlled trial.

The double oblique approach undoubtedly presents limitations. As for all double oblique approaches, it is certainly more technically challenging than using pure axial accesses. $^{12}$ It might be difficult to perform in cases of extensive cortical destruction rendering the anatomical bony landmarks (most importantly the hip joint and the ischial spine) less discernible. Finally, the very anterior part of the acetabulum is not easily covered by this approach. In such cases, an additional anterior needle might be required to optimise filling of the supra-acetabular region.

\section{References}

1. Cotten A, Deprez X, Migaud H, et al. Malignant acetabular osteolyses: percutaneous injection of acrylic bone cement. Radiology 1995 Oct;197(1):307 10.

2. Garnon J, Meylheuc L, Cazzato RL, et al. Percutaneous extra spinal cementoplasty in patients with cancer: a systematic review of proce dural details and clinical outcomes. Diagn Interv Imag 2019 Dec;100(12):743 52. https://doi.org/10.1016/j.diii.2019.07.005.

3. Durfee RA, Sabo SA, Letson GD, et al. Percutaneous acetabuloplasty for metastatic lesions to the pelvis. Orthopedics 2017 Jan1;40(1):e170 5. https://doi.org/10.3928/01477447 2016101705.

4. Dodré E, Lefebvre G, Cockenpot E, et al. Interventional MSK procedures: the hip. Br J Radiol 2016;89(1057):20150408. https://doi.org/10.1259/ bjr.20150408.

5. Oñate Miranda M, Moser TP. A practical guide for planning pelvic bone percutaneous interventions (biopsy, tumour ablation and cemento plasty). Insights Imaging 2018 Jun;9(3):275 85. https://doi.org/10.1007/ s13244 $0180600 \mathrm{y}$.

6. Hesler MC, Buy X, Catena V, et al. Assessment of risk factors for occur rence or worsening of acetabular fracture following percutaneous cementoplasty of acetabulum malignancies. Eur J Radiol 2019 Nov;120:108694. https://doi.org/10.1016/j.ejrad.2019.108694.

7. Moser TP, Onate M, Achour K, et al. Cementoplasty of pelvic bone me tastases: systematic assessment of lesion filling and other factors that could affect the clinical outcomes. Skeletal Radiol 2019;48(9):1345 55. https://doi.org/10.1007/s00256 01931560.

8. Garnon J, Meylheuc L, Auloge P, et al. Continuous injection of large volumes of cement through a single 10G vertebroplasty needle in cases of large osteolytic lesions. Cardiovasc Interv Radiol 2020 Apr;43(4):658 61. https://doi.org/10.1007/s00270 019023891.

9. Deschamps F, Yevich S, Gravel G, et al. Percutaneous fixation by internal cemented screw for the treatment of unstable osseous disease in cancer patients. Semin Interv Radiol 2018;35(4):238 47. https://doi.org/ $10.1055 / \mathrm{s} 00381673359$.

10. Yevich S, Tselikas L, Gravel G, et al. Percutaneous cement injection for the palliative treatment of osseous metastases: a technical review. Semin Interv Radiol 2018;35(4):268 80. https://doi.org/10.1055/s 00381673418.

11. Tselikas L, Joskin J, Roquet F, et al. Percutaneous bone biopsies: com parison between flat panel cone beam CT and CT scan guidance. Car diovasc Interv Radiol 2015 Feb;38(1):167 76. https://doi.org/10.1007/ s00270 01408709.

12. Sato R, Aramaki T, Yoza K, et al. "Direct MPR”: a useful tool for oblique CT fluoroscopy assisted puncture. Cardiovasc Interv Radiol 2017 Aug;40(8):1261 6. https://doi.org/10.1007/s00270 01716420.

13. Pugh R, Wessel B, Zuberi OS, et al. Iliac bone biopsy complicated by hematoma: hemostasis achieved with glue injection. Radiol Case Rep 2018 Sep 13;13(6):1112 5. https://doi.org/10.1016/j.radcr.2018.08.005.

14. Huang AJ, Halpern EF, Rosenthal DI. Incidence of delayed complications following percutaneous CT guided biopsy of bone and soft tissue lesions of the spine and extremities: a 2 year prospective study and analysis of risk factors. Skeletal Radiol 2013 Jan;42(1):61 8. https://doi.org/10.1007/ s00256 01214332.

15. Dean JR, Ison KT, Gishen P. The strengthening effect of percutaneous vertebroplasty. Clin Radiol 2000;55(6):471 6. https://doi.org/10.1053/ crad.2000.0478. 